\title{
NEGLIGEE LUXATION OF THE CARPO-METACARPIANA DORSUM OF THE FIFTH FINGER WITHOUT FRACTURE ASSOCIATED: ABOUT A CASE
}

\author{
J.Amghar, M. Yahyaoui, M. Benhammou, S. Aharram, O. Agoumi, A. Daoudi
}

Orthopedic Trauma Department A, Mohammed VI University Hospital, Faculty of Medicine and Pharmacy, Mohammed Premier University, Oujda, Morocco.

\section{ABSTRACT}

Traumatic carpal-metacarpal dislocations without associated fracture are rare lesions and their dorsal component is exceptional. Untreated, these lesions can lead to joint instability and early joint degeneration. We report the case of a dislocated dislocation of the dorsal carpo-metacarpal of the fifth metacarpal in a 26-year-old man. On clinical examination, the patient had a rotational defect with a limitation of adduction (radial inclination). The imaging assessment revealed a diastasis between the base of the fourth and fifth metacarpals, accompanied by the dorsal dislocation of the base of the fifth metacarpal. The treatment consisted of an open reduction maintained by racking. The joint was immobilized for 6 weeks. At 14 months of follow-up, the patient presented no pain and had resumed all her activities without any embarrassment.

Keywords: Carpo-metacarpal dislocation; dorsal; neglected, open hearth

${ }^{*}$ Correspondence to Author:

J.Amghar

Orthopedic Trauma Department A, Mohammed VI University Hospital, Faculty of Medicine and Pharmacy, Mohammed Premier University, Oujda, Morocco.

How to cite this article:

J.Amghar, M. Yahyaoui, M. Benhammou, S. Aharram, O. Agoumi, A. Daoudi.NEGLIGEE LUXATION OF THE CARPO-METACARPIANA DORSUM OF THE FIFTH FINGER WITHOUT FRACTURE ASSOCIATED: ABOUT A CASE. American Journal of Orthopedic Research and Reviews, 2019, 2:8

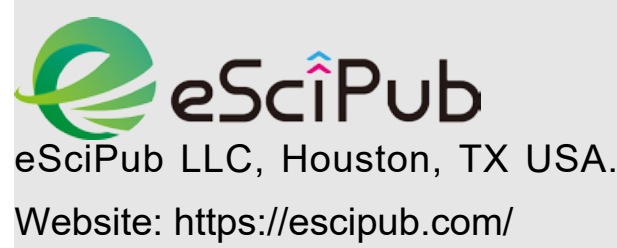




\section{Introduction}

Carpo-metacarpal dislocations of the fingers are rare lesions. The first case was described by Rivington in 1873 [1]. Occur most often as a result of high energy trauma in young adults.

The mechanism of displacement, palmar or dorsal, is due to a violent force perpendicular to the head of the metacarpal. The diagnosis is not always initially made because of the apparent normality of X-rays. The recommended treatment is closed reduction, followed or not by a racking and immobilization splint.

Sometimes an open-focus reduction with internal fixation is necessary, especially in the case of delayed diagnosis. Invasive carpometacarpal dislocation is responsible for persistent pain as well as weakness of grasping [2], which may require arthrodesis, which is often poorly tolerated. The aim of this article is to report a case of a very rare lesion, the diagnosis of which requires a thorough clinical examination and a thorough radiological analysis.

\section{Patient and Observation}

We report the case of a 26-year-old man, manual worker, right-handed, with no notable pathological antecedents. The history of the disease dates back to 2 months of admission when the patient suffered a closed trauma of the left hand following a stroke of irritation gives a blow fist against the wall causing him pain and total functional impotence of the left hand. Initially treated by Jbira for 15 days.

Given the lack of improvement and the presence of deformity on the back of the left hand, the patient consulted our structure for support. Clinical examination revealed edema and deformity of the dorsal surface of the left hand (Figure 1). The frontal and lateral radiographs (Figure 2) of the left hand as well as the CT with $3 \mathrm{D}$ reconstruction (Figure 3 ), highlighting the dorsal dislocation of the base of the 5th metacarpal, both at the level of the hamatometacarpal joint than at the level of the articulation between the bases of the 4th and 5th metacarpals, without associated fracture of the carp bones.

Under locoregional anesthesia and scopic control, an open-focus reduction was performed, (Figure 4) by a medial incision taken off in dorsal junction between palmar and dorsal skin, then passage between the extensor tendon of the 5 th finger and posterior ulnar tendon, a capsulotomy was performed with a resection of fibrosis (a good condition of the articular cartilage was observed), reduction with a jump was felt and heard, and the reduction seemed stable. As a precaution, however, the patient benefited from carpo-metacarpal insertion with two Kirschner wires (Figure 4). No ligament repair was performed.

An immobilization by antebrachio-palmar splint was put in place for six weeks with elevation of the hand and active mobilization of the fingers. The postoperative radiological examinations objectified the perfect reduction of the dislocation (figure 5). At 12 months of follow-up, the patient was seen in pain-free consultation and had complete functional recovery with normal activity recovery, normal finger coil, unrestricted and symmetrical range of motion, and an excellent DASH score of 0.83 .

\section{Discussion}

The carpo-metacarpal joints are particularly stable because of the convergence on the metacarpal base of the intermetacarpal ligaments, the palmar carpo-metacarpal and the carpo-metacarpal dorsal [3]. Only very violent trauma can cause carpo-metacarpal dislocation.

This rare lesion accounts for less than $1 \%$ of all wrist and carp trauma [4]. The radiological diagnosis can easily be missed on ordinary pictures, especially if there is no associated fracture. Diagnosis can be facilitated by performing dynamic radiography [5] by applying compression or traction.

The interpretation of ordinary X-rays is not easy. Classically, displacement of the 5th metacarpal with respect to the hamatum is observed, with loss of alignment and overlap. Clinical 
J.Amghar et al., AJORR, 2019 2:8

examination reveals pain, deformity of the hand young adult following a two-wheeled accident, and functional impotence. Carpo-metacarpal causing a violent shock of the metacarpal on the dislocations are usually dorsal, most often handlebars [6]. associated with fractures and typically affect the

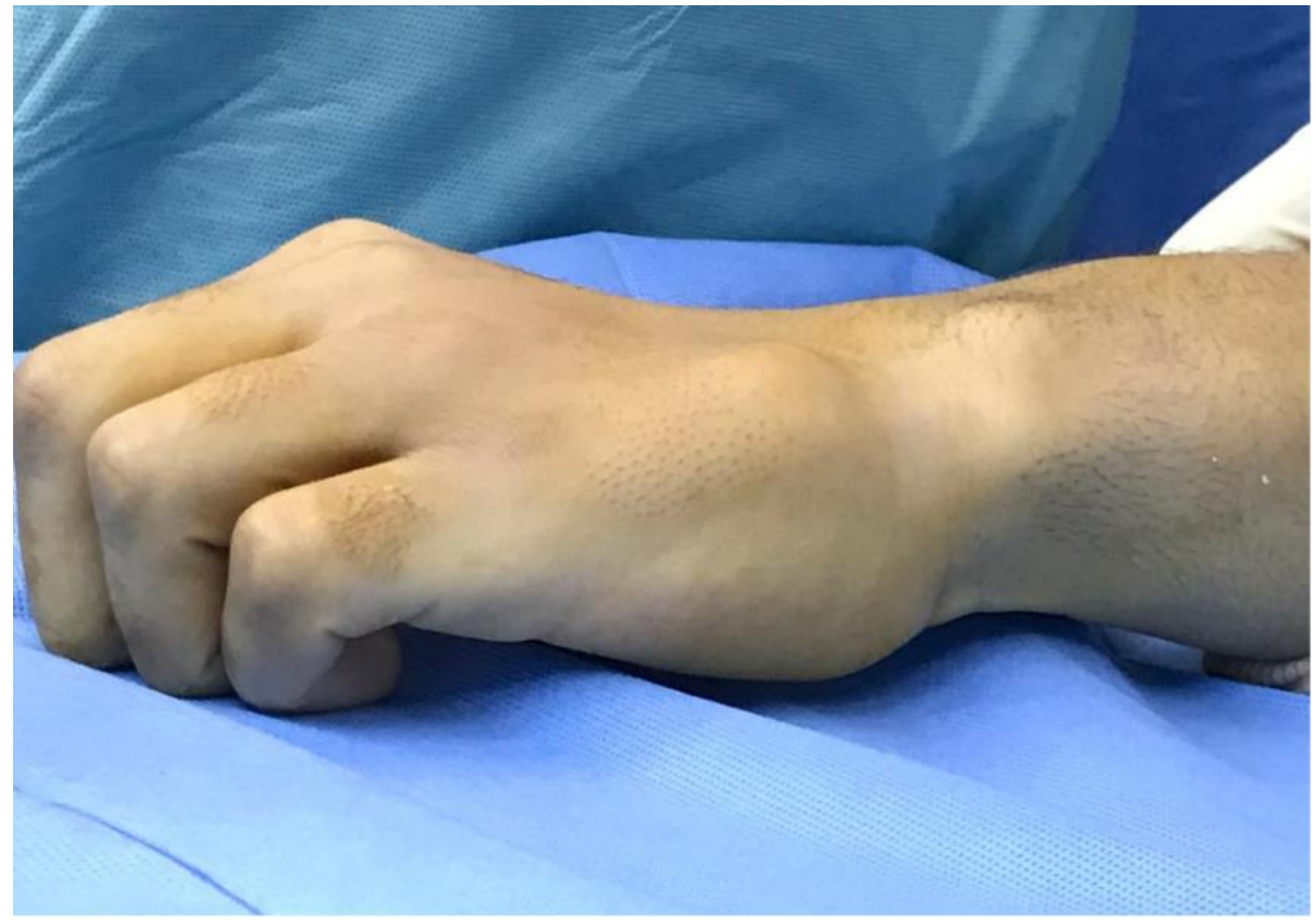

Figure 1 : Image showing edema and deformity of the dorsal surface of the left hand

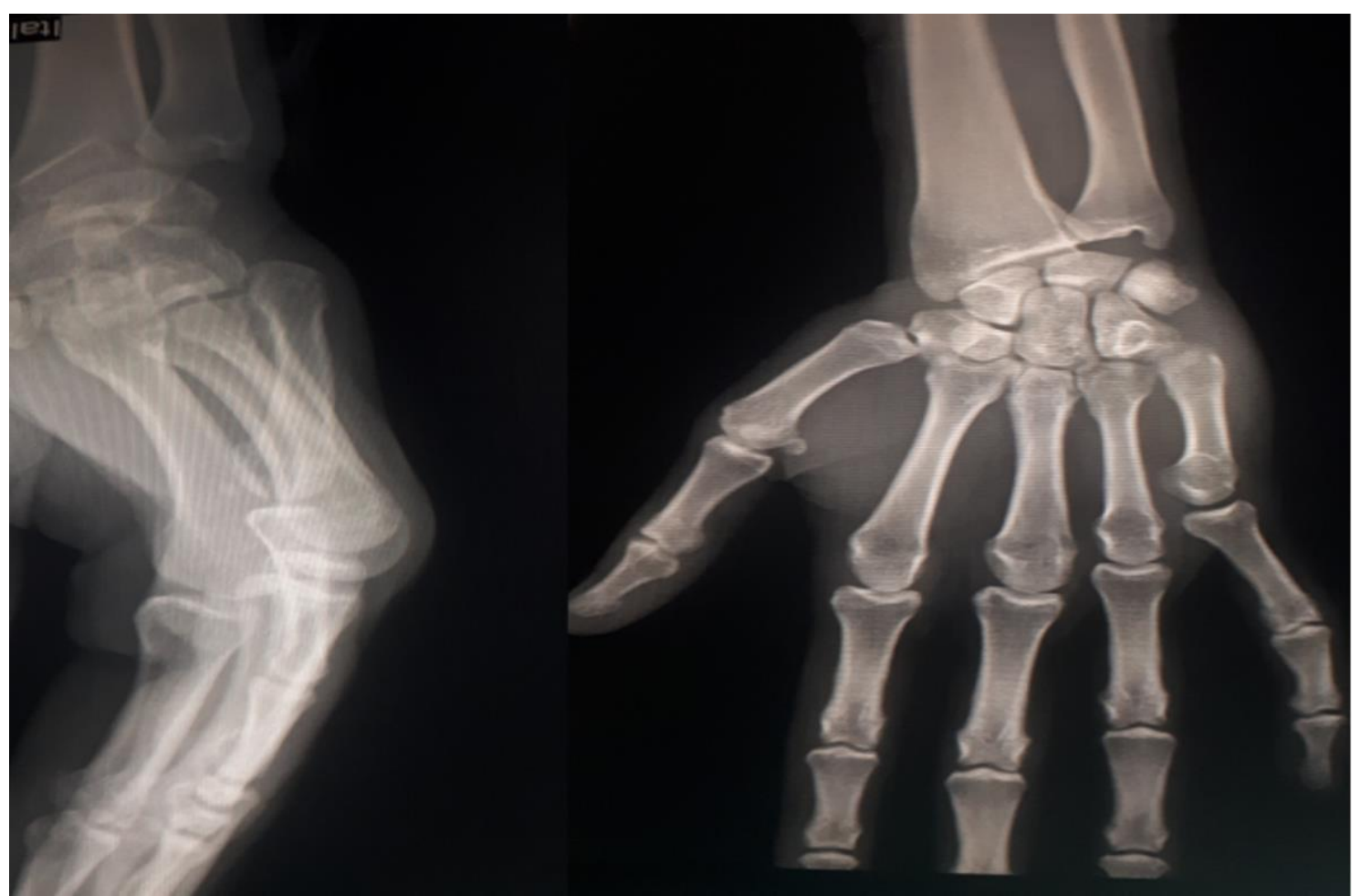

Figure 2: x-ray of face and profile showing a luxation of the carpo-metacarpienne dorsale of the fifth finger

.AJORR: https://escipub.com/american-journal-of-orthopedic-research-and-reviews/ 3 


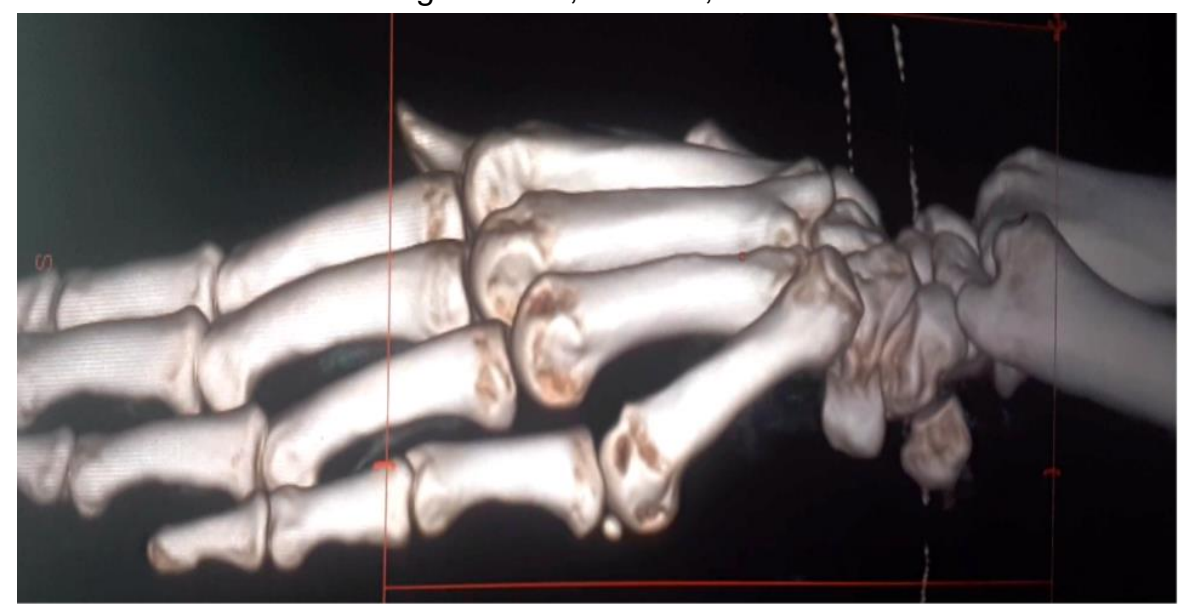

Figure 3: CT with 3D reconstruction

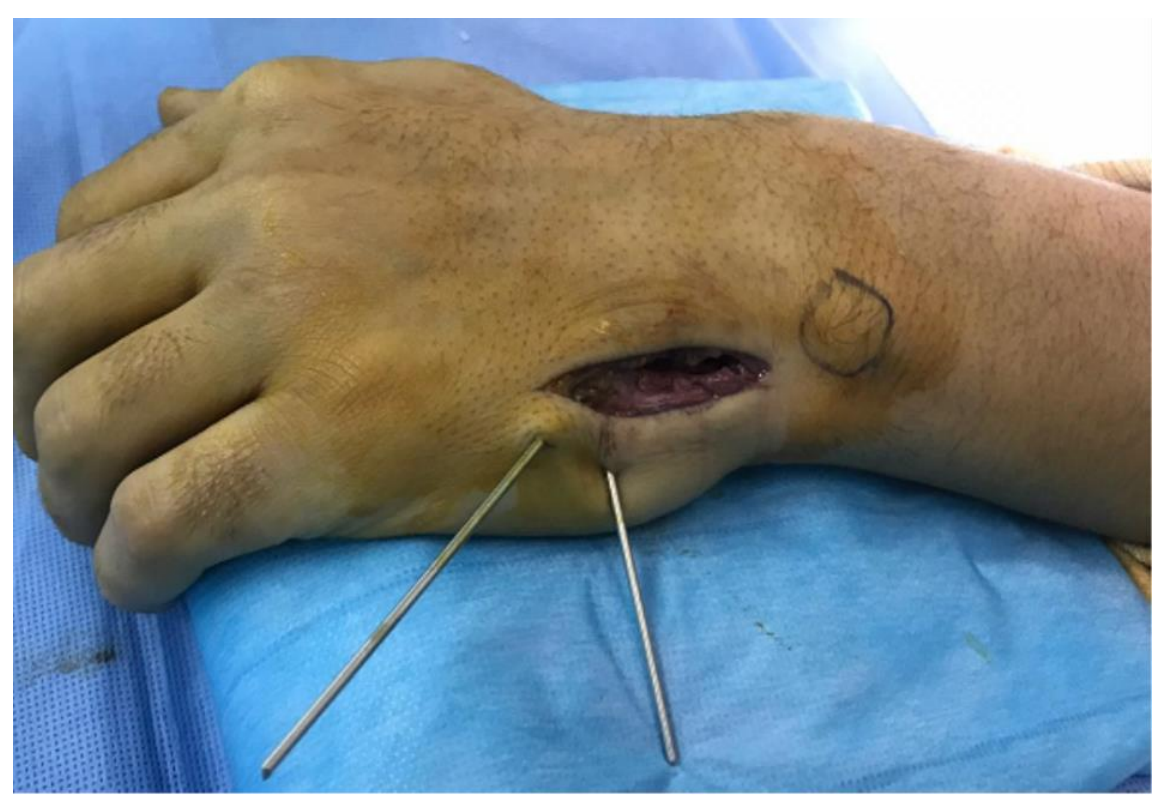

Figure 4: Per-operative image showing Carpo-metacarpal insertion by two Kirschner pins
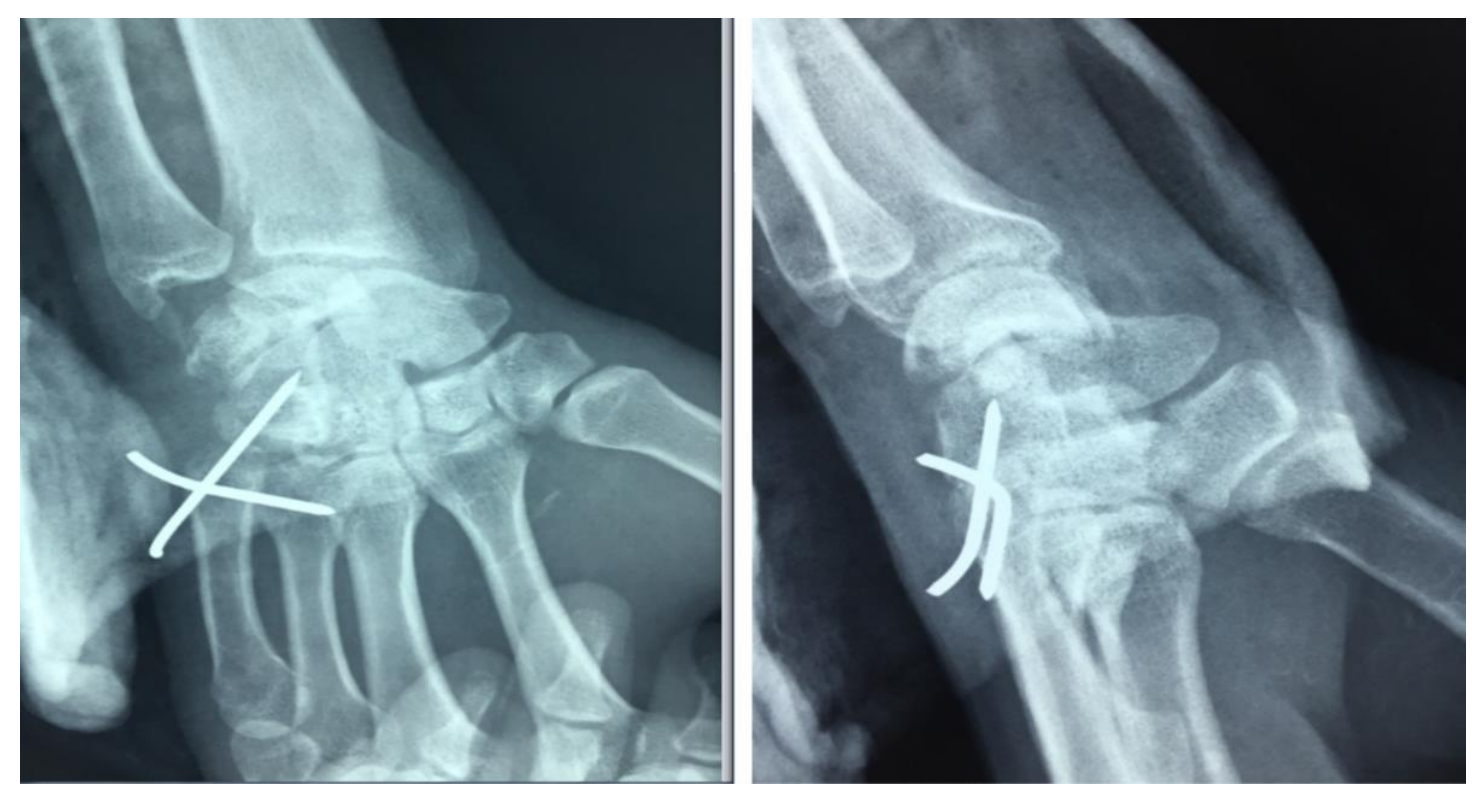

Figure 5: Postoperative radiological examinations showed the perfect reduction of dislocation 
We distinguish the direct mechanism when the force is applied directly on the base of the metacarpals causing a dislocation dorsal or palmar in the direction of the vector, the indirect mechanism when the force is applied opposite the head of the metacarpal, causing a dislocation of its based. The classification of Costagliola according to the seat, the number and the displacement is the most used and individualizes two entities: the columnar axis for the column of the thumb and the spatular axis for the long fingers. Surgical treatment is often necessary, including closed-focus reduction (or open-air reduction if the reduction is not anatomical) followed by carpo-metacarpal insertion.

Postoperative immobilization is generally recommended for 4 to 6 weeks. Carpometacarpal dislocations can have serious consequences if they are not treated adequately, hence the importance of early diagnosis. The deep branch of the ulnar nerve passing next to the 5th carpo-metacarpal joint is vulnerable to both dorsal [7] and palmar dislocations [8]. The prognosis is often good, but there is a risk of persistent pain and limitation of movement.

\section{Conclusion}

Carpo-metacarpal dislocations of the long fingers are rare lesions that deserve to be highlighted because their diagnosis is often delayed, often associated with fractures of the carp or the bones of the hand. Many go unnoticed either because of a summary exam that is poorly done or falls within the framework of a polytrauma. Subject to urgent and correct treatment, they are of good prognosis

\section{References}

1. Kneife F. Simultaneous dislocations of the five carpometacarpal joints. Injury. 2002 Nov; 33(9):846. PubMed | Google Sc

2. Gore DR. Carpometacarpal dislocation producing compression of the deep branch of the ulnar nerve. J Bone Joint Surg Am. 1971 oct; 53(7):1387-90. PubMed | Google Scholar.
3. Nalebuff EA. Isolated anterior carpometacarpal dislocation of the fifth finger: classification and case report. J Trauma 1968;8:1119-23:

4. Dobyns JH, Linscheid RL, Cooney WP 3rd. Fractures and dislocations of 225 the wrist and hand, then and now. J Hand Surg Am 1983;8:687-90

5. Yamakado K, Hashimoto F, Nagata S, Higuchi M. Isolated palmar 217 dislocation of the fifth carpometacarpal joint diagnosed by stress $\mathrm{X}$-rays. 218 Arch Orthop Trauma Surg 2000;120:529-30.

6. Diez E, Marti D, Aramburo F, Mendez JM. Multiple carpo-metacarpal 227 dislocations. Five cases. Ann Chir Main

7. Young TB. Dorsal dislocation of the metacarpal base of the little and ring 220 fingers with ulnar nerve branch compression. Injury 1987;18:65-6

8. Gore DR. Carpo-metacarpal dislocation producing compression of the 170 deep branch of the ulnar nerve. J Bone Joint Surg Am 1971;53:1387-90.

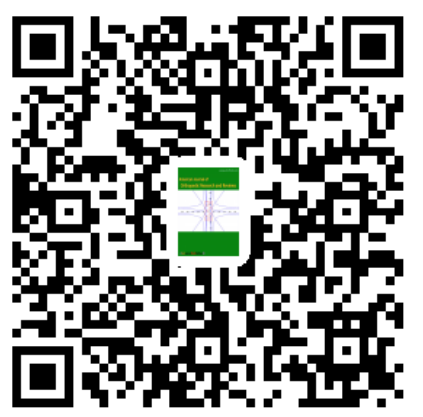

